\title{
A Significance of High Prevalence of Diabetes and Hypertension in Severe COVID-19 Patients
}

\author{
Hidekatsu Yanai
}

\section{To the Editor}

Severe acute respiratory syndrome coronavirus 2 (SARSCoV-2), which causes coronavirus disease 2019 (COVID-19) has reached a pandemic level. In a recent retrospective cohort study of COVID-19, comorbidities such as diabetes and hypertension were present in nearly half of patients [1]. The odds of in-hospital death were significantly higher in patients with diabetes (2.85) and hypertension (3.05) [1].

It remains unknown why the prevalence of diabetes and hypertension is high in COVID-19 patients and why such diseases can be risk for severe COVID-19 including death.

To elucidate such clinical questions, I searched the prevalence of diabetes and hypertension and the controlling status of such diseases in all COVID-19 patients, severe and nonsevere COVID-19 patients using PubMed. The prevalence of each disease in severe and non-severe COVID-19 patients was meta-analyzed by Peto's exact method, and odds ratio (OR) with $95 \%$ confidence interval $(\mathrm{CI})$ was shown.

The prevalence of diabetes in all COVID-19 patients, severe and non-severe COVID-19 patients were shown in Table 1 [1-12]. Half of studies showed a statistically significantly higher prevalence of diabetes in severe patients than in non-severe patients. In the meta-analysis, the prevalence of diabetes in severe patients was significantly higher than that in non-severe patients (OR: 3.52 , 95\% CI: 2.65 - 4.67). Unfortunately, only three studies were available to analyze the controlling status of diabetes. All three studies showed that plasma glucose levels in severe patients were significantly higher than those in non-severe patients. Although such data did not present sufficient evidence, elevation of plasma glucose is likely to be associated with severity of COVID-19. Zhu et al performed a retrospective, multicentered study of 7,337 cases of COVID-19 in Hubei Province, China, among which 952 had pre-existing type 2 diabetes [13]. They found that subjects with type 2 diabetes required more medical interventions, and had a significantly higher mortality ( $7.8 \%$ vs. $2.7 \%)$ and multiple organ injury than the non-diabetic

Manuscript submitted May 15, 2020, accepted May 26, 2020

Published online June 4, 2020

Department of Diabetes, Endocrinology and Metabolism, National Center for Global Health and Medicine Kohnodai Hospital, 1-7-1 Kohnodai, Ichikawa, Chiba 272-8516, Japan. Email: dyanai@hospk.ncgm.go.jp

doi: https://doi.org/10.14740/jocmr4218 individuals. Further, well-controlled blood glucose was associated with markedly lower mortality compared to individuals with poorly controlled blood glucose, supporting a significance of hyperglycemia in severe COVID-19.

The prevalence of hypertension in all COVID-19 patients, severe and non-severe COVID-19 patients was shown in Table 2 [1-12]. Five of 12 studies showed a statistically significantly higher prevalence of hypertension in severe patients than in non-severe patients. In the meta-analysis, the prevalence of hypertension in severe patients was significantly higher than that in non-severe patients (OR: 2.69, 95\% CI: 2.16 - 3.34). Seven studies were available to analyze the controlling status of hypertension. Five studies did not show any difference in blood pressure between severe and non-severe patients. Huang et al showed that systolic blood pressure was significantly higher in severe patients than in non-severe patients [2]. Itelman et al showed that minimal systolic blood pressure was significantly lower in severe patients than in non-severe patients, and maximal systolic blood pressure was significantly higher in severe patients than in non-severe patients [11]. Blood pressure itself is unlikely to be associated with severity of COVID-19. Since hypertension is one of the most significant risk factors for severe disease and mortality in COVID-19, and angiotensin-converting enzyme (ACE) 2 is the entry receptor of SARS-CoV-2 [14, 15], the effects of ACE inhibitors (ACEIs) and angiotensin receptor blockers (ARBs) in COVID-19 patients have received much focus. A recent study has shown the protective effects of ACEIs/ ARBs against mortality in COVID-19 [16], challenging the association between ACEIs/ARBs use and severe COVID-19.

Why is hypertension one of the most significant risk factors for severe disease and mortality in COVID-19? Diabetes and hypertension are crucial coronary risk factors, and diabetes and hypertension are closely interlinked because of similar risk factors, such as endothelial dysfunction, vascular inflammation, arterial re-modelling, atherosclerosis, dyslipidemia, and obesity [17]. Common mechanisms, such as upregulation of the reninangiotensin-aldosterone system, oxidative stress, inflammation, and activation of the immune system likely contribute to the close relationship between diabetes and hypertension [17]. High prevalence of diabetes and hypertension in severe COVID-19 patients can be explained by such vascular damage. The prevalence of cardiovascular disease (CVD) in all COVID-19 patients, severe and non-severe COVID-19 patients was shown in Table $3[1-8,10-12]$. Four of 11 studies showed a statistically significantly higher prevalence of CVD in severe patients than in non-severe patients. In the meta-analysis, the prevalence of 
Table 1. Prevalence of Diabetes in COVID-19 Patients

\begin{tabular}{lllll}
\hline & All patients & Severe patients & Non-severe patients & P value \\
\hline Huang et al, 2020 [2] & $8 / 41(20 \%)$ & $1 / 13(8 \%)$ & $7 / 28(25 \%)$ & 0.16 \\
Zhang et al, 2020 [3] & $17 / 140(12.1 \%)$ & $8 / 58(13.8 \%)$ & $9 / 82(11 \%)$ & 0.615 \\
Wang et al, 2020 [4] & $14 / 138(10.1 \%)$ & $8 / 36(22.2 \%)$ & $6 / 102(5.9 \%)$ & 0.009 \\
Wu et al, 2020 [5] & $22 / 201(10.9 \%)$ & $16 / 84(19 \%)$ & $6 / 117(5.1 \%)$ & 0.002 \\
Zhou et al, 2020 [1] & $36 / 191(19 \%)$ & $17 / 54(31 \%)$ & $19 / 137(14 \%)$ & $0 \cdot 0051$ \\
Wan et al, 2020 [6] & $12 / 135(8.9 \%)$ & $9 / 40(22.5 \%)$ & $3 / 95(3.1 \%)$ & NA \\
Guan et al, 2020 [7] & $30 / 1,590(8.2 \%)$ & $19 / 130(14.6 \%)$ & $80 / 1,460(5.5 \%)$ & 0.116 \\
Wang et al, 2020 [8] & $54 / 339(16 \%)$ & $11 / 65(17.2 \%)$ & $43 / 274(15.8 \%)$ & 0.08 \\
Chen et al, 2020 [9] & $14 / 145(9.7 \%)$ & $7 / 43(16.3 \%)$ & $7 / 102(6.9 \%)$ & $<0.001$ \\
Hu et al, 2020 [10] & $47 / 323(14.6 \%)$ & $11 / 26(42.3 \%)$ & $14 / 151(9.3 \%)$ & 0.04 \\
Itelman et al, 2020 [11] & $30 / 162(18.5 \%)$ & $11 / 92(30.8 \%)$ & $8 / 26(12 \%)$ & $11 / 179(6.1 \%)$ \\
Huang et al, 2020 [12] & $19 / 202(9.4 \%)$ & $8 / 23(34.8 \%)$ & $<0.001$ \\
\hline
\end{tabular}

$P$ values indicate a statistically significant difference between severe and non-severe patients. NA: not available; COVID-19: coronavirus disease 2019.

CVD in severe patients was significantly higher than that in non-severe patients (OR: 5.37, 95\% CI: 3.73 - 7.74). Further, CVD was significantly associated with increased odds of death (OR: 21.4) [1], supporting a significant association between vascular damage and severity of COVID-19.

D-dimer is detected in injured vascular endothelium [18], and von Willebrand factor is produced by endothelial cells in response to injury, and high von Willebrand factor level has been observed in systemic vasculitis [19]. Multivariable regression showed increasing odds of in-hospital death associated with Ddimer greater than $1 \mu \mathrm{g} / \mathrm{mL}$ (OR: 18.42, 95\% CI: 2.64 - 128.55; $\mathrm{P}=0.0033$ ) on admission [1]. Elevated von Willebrand factor levels were observed in COVID-19 patients in the intensive care unit [20]. D-dimer was also reported to be significantly increased in Kawasaki disease with coronary artery lesions, and to be the independent risk for coronary artery lesions in Kawasaki disease [21]. Von Willebrand factor parameters were reported to represent potential biomarkers for disease activity and coronary artery lesion in patients with Kawasaki disease [22]. A very recent article reported the development of Kawasaki disease in COVID-19 patient [23], supporting an association between endothelial injury and severe COVID-19.

In conclusion, endothelial injury may be associated with severity of COVID-19, which can partially explain high prevalence of diabetes and hypertension in severe COVID-19.

\section{Acknowledgments}

I thank the staffs (Yukie Kawamura, Keiko Nakamura, Harue

Table 2. Prevalence of Hypertension in COVID-19 Patients

\begin{tabular}{lllll}
\hline & All patients & Severe patients & Non-severe patients & P value \\
\hline Huang et al, 2020 [2] & $6 / 41(15 \%)$ & $2 / 13(15 \%)$ & $4 / 28(14 \%)$ & 0.93 \\
Zhang et al, 2020 [3] & $42 / 140(30 \%)$ & $22 / 58(37.9 \%)$ & $20 / 82(24.4 \%)$ & 0.085 \\
Wang et al, 2020 [4] & $43 / 138(31.2 \%)$ & $21 / 36(58.3 \%)$ & $22 / 102(21.6 \%)$ & $<0.001$ \\
Wu et al, 2020 [5] & $39 / 201(19.4 \%)$ & $23 / 84(27.4 \%)$ & $16 / 117(13.7 \%)$ & 0.02 \\
Zhou et al, 2020 [1] & $58 / 191(30 \%)$ & $26 / 54(48 \%)$ & $32 / 137(23 \%)$ & $0 \cdot 0008$ \\
Wan et al, 2020 [6] & $13 / 135(9.6 \%)$ & $4 / 40(10 \%)$ & $9 / 95(9.4 \%)$ & NA \\
Guan et al, 2020 [7] & $269 / 1,590(16.9 \%)$ & $38 / 269(14.1 \%)$ & $61 / 1,321(4.6 \%)$ & $106 / 274(38.8 \%)$ \\
Wang et al, 2020 [8] & $138 / 339(40.8 \%)$ & $32 / 65(50 \%)$ & $13 / 102(12.7 \%)$ & 0.031 \\
Chen et al, 2020 [9] & $22 / 145(15.2 \%)$ & $9 / 43(20.9 \%)$ & $39 / 151(25.8 \%)$ & 0.21 \\
Hu et al, 2020 [10] & $105 / 323(32.5 \%)$ & $10 / 26(38.5 \%)$ & $18 / 92(19.6 \%)$ & 0.056 \\
Itelman et al, 2020 [11] & $49 / 162(30.2 \%)$ & $13 / 26(50 \%)$ & $27 / 179(15.1 \%)$ & 0.002 \\
Huang et al, 2020 [12] & $29 / 202(14.4 \%)$ & $2 / 23(8.7 \%)$ & 0.411 \\
\hline
\end{tabular}

$P$ values indicate a statistically significant difference between severe and non-severe patients. NA: not available; COVID-19: coronavirus disease 2019. 
Table 3. Prevalence of Cardiovascular Disease in COVID-19 Patients

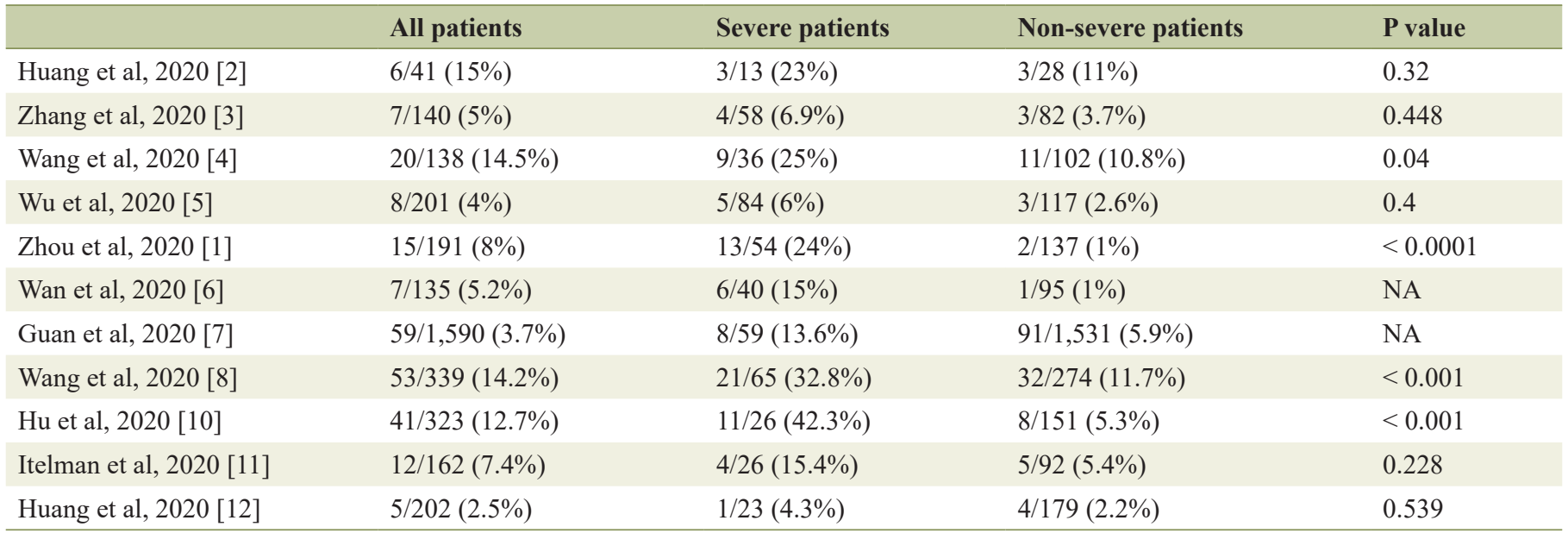

$P$ values indicate a statistically significant difference between severe and non-severe patients. NA: not available; COVID-19: coronavirus disease 2019.

Aoki and Ayano Sakakibara) of the Division of Research Support, National Center for Global Health and Medicine Kohnodai Hospital.

\section{Financial Disclosure}

Author has no financial disclosures to report.

\section{Conflict of Interest}

The author declares that he has no conflict of interest concerning this article.

\section{Informed Consent}

Not applicable.

\section{Author Contributions}

HY designed the research, collected and analyzed data, and wrote and approved the final paper.

\section{Data Availability}

The author declares that data supporting the findings of this study are available within the article.

\section{References}

1. Zhou F, Yu T, Du R, Fan G, Liu Y, Liu Z, Xiang J, et al. Clinical course and risk factors for mortality of adult inpatients with COVID-19 in Wuhan, China: a retrospective cohort study. Lancet. 2020;395(10229):1054-1062.

2. Huang C, Wang Y, Li X, Ren L, Zhao J, Hu Y, Zhang $\mathrm{L}$, et al. Clinical features of patients infected with 2019 novel coronavirus in Wuhan, China. Lancet. 2020;395(10223):497-506.

3. Zhang JJ, Dong X, Cao YY, Yuan YD, Yang YB, Yan YQ, Akdis CA, et al. Clinical characteristics of 140 patients infected with SARS-CoV-2 in Wuhan, China. Allergy. 2020.

4. Wang D, Hu B, Hu C, Zhu F, Liu X, Zhang J, Wang B, et al. Clinical Characteristics of 138 Hospitalized Patients With 2019 Novel Coronavirus-Infected Pneumonia in Wuhan, China. JAMA. 2020.

5. Wu C, Chen X, Cai Y, Xia J, Zhou X, Xu S, Huang H, et al. Risk Factors Associated With Acute Respiratory Distress Syndrome and Death in Patients With Coronavirus Disease 2019 Pneumonia in Wuhan, China. JAMA Intern Med. 2020.

6. Wan S, Xiang Y, Fang W, Zheng Y, Li B, Hu Y, Lang C, et al. Clinical features and treatment of COVID-19 patients in northeast Chongqing. J Med Virol. 2020.

7. Guan WJ, Liang WH, Zhao Y, Liang HR, Chen ZS, Li YM, Liu XQ, et al. Comorbidity and its impact on 1590 patients with COVID-19 in China: a nationwide analysis. Eur Respir J. 2020;55(5).

8. Wang L, He W, Yu X, Hu D, Bao M, Liu H, Zhou J, et al. Coronavirus disease 2019 in elderly patients: Characteristics and prognostic factors based on 4-week follow-up. J Infect. 2020;80(6):639-645.

9. Chen Q, Zheng Z, Zhang C, Zhang X, Wu H, Wang J, Wang S, et al. Clinical characteristics of 145 patients with corona virus disease 2019 (COVID-19) in Taizhou, Zhejiang, China. Infection. 2020.

10. Hu L, Chen S, Fu Y, Gao Z, Long H, Wang JM, Ren HW, et al. Risk factors associated with clinical outcomes in 323 COVID-19 hospitalized patients in Wuhan, China. Clin Infect Dis. 2020. 
11. Itelman E, Wasserstrum Y, Segev A, Avaky C, Negru L, Cohen D, Turpashvili N, et al. Clinical characterization of 162 COVID-19 patients in Israel: preliminary report from a large tertiary center. Isr Med Assoc J. 2020;22(5):271274.

12. Huang R, Zhu L, Xue L, Liu L, Yan X, Wang J, Zhang B, et al. Clinical findings of patients with coronavirus disease 2019 in Jiangsu province, China: A retrospective, multicenter study. PLoS Negl Trop Dis. 2020;14(5):e0008280.

13. Zhu L, She ZG, Cheng X, Qin JJ, Zhang XJ, Cai J, Lei F, et al. Association of blood glucose control and outcomes in patients with COVID-19 and pre-existing type 2 Diabetes. Cell Metab. 2020.

14. Cure E, Cumhur Cure M. Angiotensin-converting enzyme inhibitors and angiotensin receptor blockers may be harmful in patients with diabetes during COVID-19 pandemic. Diabetes Metab Syndr. 2020;14(4):349-350.

15. Vaduganathan M, Vardeny O, Michel T, McMurray JJV, Pfeffer MA, Solomon SD. Renin-angiotensin-aldosterone system inhibitors in patients with COVID-19. N Engl J Med. 2020;382(17):1653-1659.

16. Zhang P, Zhu L, Cai J, Lei F, Qin JJ, Xie J, Liu YM, et al. Association of inpatient use of angiotensin converting enzyme inhibitors and angiotensin II receptor blockers with mortality among patients with hypertension hospitalized with COVID-19. Circ Res. 2020.

17. Petrie JR, Guzik TJ, Touyz RM. Diabetes, hypertension, and cardiovascular disease: clinical insights and vascular mechanisms. Can J Cardiol. 2018;34(5):575-584.

18. Kaplan KL, Bini A, Fenoglio J, Jr., Kudryk B. Fibrin and the vessel wall. Adv Exp Med Biol. 1990;281:313-318.

19. Bleil L, Manger B, Winkler TH, Herrman M, Burmester GR, Krapf FE, Kalden JR. The role of antineutrophil cytoplasm antibodies, anticardiolipin antibodies, von Willebrand factor antigen, and fibronectin for the diagnosis of systemic vasculitis. J Rheumatol. 1991;18(8):11991206.

20. Panigada M, Bottino N, Tagliabue P, Grasselli G, Novembrino C, Chantarangkul V, Pesenti A, et al. Hypercoagulability of COVID-19 patients in intensive care unit. A report of thromboelastography findings and other parameters of hemostasis. J Thromb Haemost. 2020.

21. Kong WX, Ma FY, Fu SL, Wang W, Xie CH, Zhang YY, Gong FQ. Biomarkers of intravenous immunoglobulin resistance and coronary artery lesions in Kawasaki disease. World J Pediatr. 2019;15(2):168-175.

22. Jakob A, Schachinger E, Klau S, Lehner A, Ulrich S, Stiller B, Zieger B. Von Willebrand factor parameters as potential biomarkers for disease activity and coronary artery lesion in patients with Kawasaki disease. Eur J Pediatr. 2020;179(3):377-384.

23. Jones VG, Mills M, Suarez D, Hogan CA, Yeh D, Segal JB, Nguyen EL, et al. COVID-19 and Kawasaki Disease: novel virus and novel case. Hosp Pediatr. 2020. 